\title{
Um Ambiente para Modelagem Organizacional Baseado em Regras de Negócio
}

Felipe G. Dias ${ }^{1}$, Gisele P. Morgado ${ }^{1}$, Alissandra E. Martins ${ }^{1}$, Célia M. Seabra ${ }^{1}$, Denis S. Silveira ${ }^{1,2}$, Antônio J. Alencar ${ }^{1}$, Priscila M. V. Lima ${ }^{1}$, Eber A. Schmitz ${ }^{1}$

${ }^{1}$ Núcleo de Computação Eletrônica - Instituto de Matemática

Universidade Federal do Rio de Janeiro (UFRJ)

Caixa Postal 2324 - 20.001-970 - Rio de Janeiro - RJ - Brasil

${ }^{2}$ Departamento de Administração

Faculdades Ibmec-RJ

Av. Rio Branco $10818^{\circ}$ andar CEP: 20.040-001 - Rio de Janeiro - RJ - Brasil

$$
\text { \{felipe.dias, gmorgado\}@ufrj.br, }
$$

alissandra, celiams\}@posgrad.nce.ufrj.br, denis@ibmecrj.br,

juarez@dcc.im.ufrj.br, \{priscila, eber\}ence.ufrj.br

Abstract. This paper presents a computational environment for organizational modelling named REGULA. The approach is based on Business Rules (BRs) in order to facilitate the alignment of the specification and development of information systems to the needs of an organization. The discussion on REGULA emphasizes three points: the incorporation of the definition of objectives and goals to BRs, the guided definition of the business vocabulary with respective internal representation and the query to permissions, obligations and prohibitions. The solution to the second point enables the automatic generation of some of the Business Rules classified as "facts", while the third issue is illustrated by the query to permissions.

Resumo. Este artigo apresenta o ambiente REGULA para modelagem organizacional. A abordagem baseia-se em Regras de Negócio (RNs), o que facilita a especificação e construção de sistemas de informação mais alinhada às necessidades de uma organização. A discussão sobre o ambiente REGULA destaca três pontos: a definição integrada de objetivos e metas às RNs, a captura assistida seguida de representação interna do vocabulário do negócio e a consulta a permissões, obrigações e proibições. A solução para o segundo ponto permite a geração automática de algumas RNs do tipo "fato", enquanto que o terceiro é ilustrado pela consulta a permissões.

\section{Introdução}

Regras são familiares a todos nós na vida real - jogamos futebol através de suas regras, vivemos sob um sistema legal baseado em um conjunto de regras e estipulamos regras para os nossos filhos. O ambiente empresarial não é diferente, nele as regras não estão aperıas presentes como também são centrais para as operações básicas do seu dia-a-dia. Assim, podemos observar nos últimos anos um crescente interesse sobre o tema, reforçando a aplicabilidade de Regras de Negócio (RNs) à construção moderna de sistemas de informação. Apesar do papel fundamental que exercem, ainda não se 
chegou a um consenso sobre tópicos-chave de RNs. Dentre tais tópicos podemos citar sua captura, representação interna e visibilidade/manuseio dentro de uma organização Os dois primeiros constituem o assunto da chamada realizada pelo Object Management Group (OMG) para a definição semântica de RNs [OMG 2004]. Quanto à visibilidade/manuseio das RNs, alguns dos problemas a serem resolvidos dizem respeito à consulta a permissões, obrigações e proibições

Este trabalho tem como objetivo apresentar uma abordagem para modelagem organizacional baseada em RNs. Tal abordagem é suportada pelo ambiente REGULA, no qual destacam-se, além do mapeamento das RNs para uma representação interna computável, o apoio à definição do vocabulário do negócio e a disponibilidade de consulta acerca das permissões, obrigações e proibições. Convém ressaltar que a definição estruturada do vocabulário do negócio, constituído pelos termos, possibilita a geração automática de algumas das RNs que representam fatos sobre elementos deste vocabulário. O restante do texto encontra-se estruturado da seguinte forma: na segunda seção conceituamos modelagem organizacional destacando seus principais componentes, na terceira seção apresentamos o ambiente RÉGULA exemplificando a captura e representação interna de termos de RNs, além de tratarmos da consulta a permissões ilustrando a utilidade da incorporação de tal funcionalidade a um ambiente baseado em RNs e, finalmente, na quarta seção tecemos alguns comentários finais além de apontar para tópicos de pesquisa em andamento e de trabalho futuro

\section{Modelagem Organizacional}

De acordo com [Eriksson e Penker 2000], uma organização possui os seguintes componentes: objetivos, recursos, processos e regras. Os objetivos do negócio descrevem o que este e suas atividades devem alcançar. Para que uma atividade do negócio se encaminhe em direção a um dado objetivo, esta atividade deve ser realizada de acordo com uma ou mais metas a ele relacionadas. Segundo [Koubarakis e Plexousakis 2002], uma meta constitui em um estado do negócio. Nossa modelagem restringe esta definição para a obtenção de um valor para uma variável do negócio dentro de um determinado prazo. A fim de atingir seus objetivos, as organizações necessitam ainda de elementos constitutivos tais como pessoas, materiais, informações ou produtos. Estes recursos são utilizados ou produzidos nos/pelos processos de negócio. A eficácia dos processos depende do seu alinhamento com as RNs da organização em questão.

As Regras de Negócio podem ser definidas sob duas perspectivas: a do negócio e a de sistemas de informação [Ross 2000]. Do ponto de vista do negócio, uma regra destina-se a influenciar ou guiar o comportamento do negócio, como suporte a uma política que é formulada em função de uma oportunidade e ameaças do ambiente no qual a organização está inserida. Por estrutura do negócio pode-se considerar o modelo estático do negócio com seus dados (termos) e os relacionamentos entre eles (fatos). Já os controles (restrições) e as influências (derivações e regras habilitadoras de ação) compõem o modelo dinâmico em que se verificam os aspectos comportamentais do modelo de negócios. Segundo a perspectiva de sistemas de informação, uma regra de negócio é uma sentença que define ou qualifica algum aspecto do negócio, representando o conhecimento dos especialistas do negócio. Através das RNs é possive garantir a estrutura do negócio ou influenciar o comportamento do mesmo.
Dentre as diversas propostas de classificação de Regras de Negócio, a mais utilizada encontra-se originalmente em [BRG 1997], onde as regras são divididas nas cinco categorias mencionadas anteriormente. Os termos constituem os elementos básicos da linguagem utilizada para expressar as RNs, onde a própria definição de um termo é considerada como uma regra. Fatos descrevem a natureza ou estrutura operacional de uma organização, relacionando os termos do negócio uns aos outros. Os cálculos e derivações determinam como um conhecimento ou informação pode ser transformado em outro, através de fórmulas ou mudanças de estado. Já as restrições, conforme o nome indica, restringem algum comportamento na organização, estando relacionadas a decisões sobre quais dados podem ou não ser atualizados. As habilitadoras de ação podem ser vistas como regras dedutivas, de raciocínio encadeado à frente, representadas através de um par contendo uma condição e respectiva ação. Vale notar que tal classificação foi ligeiramente modificada em sua versão posterior [BRG $2000]$

\section{O Ambiente RÉGULA}

O processo de desenvolvimento de sistemas de informação pode ser lento e custoso, envolvendo assim uma alta probabilidade de insucesso. Segundo [Morgan 20(2)], a maioria dos problemas nesta área surge nas fases iniciais de análise e especificação, advinda de deficiências na definição dos requisitos do negócio. Com o intuito de resolver estes problemas, Tony Morgan apresenta um ideal de construção de sistemas de informação apoiado no uso de Regras de Negócio. O ambiente RÉGULA baseia-se neste ideal cujo esquema de modelagem organizacional está representado na Figura 1.

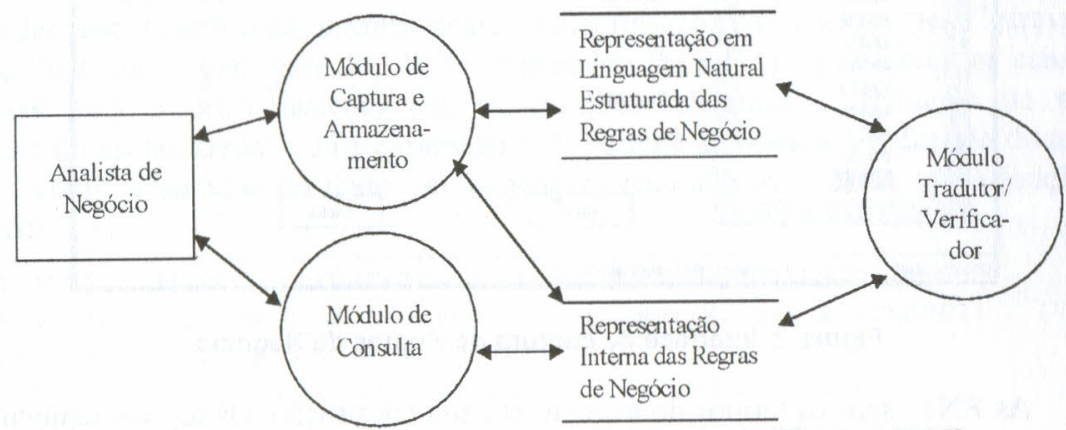

Figura 1. Baseado em fragmento da figura 1-2 de [Morgan 2002].

\subsection{Captura e Armazenamento}

A modelagem organizacional parte da especificação das necessidades da organização pelo analista de negócio. Para isso, o analista deve definir os objetivos gerais do negócio que satisfaçam a essas necessidades. Para cada objetivo, o analista deve também definir uma ou mais metas que possam guiar as atividades da organização no sentido de atingir esse objetivo. Cada meta possui um identificador distinto. Uma meta está associada a uma determinada variável do negócio, sendo também determinados o valor que esta variável deve atingir e o prazo para que isso ocorra. As variáveis do negócio devem estar definidas no RÉGULA como termos. 
Além da especificação dos objetivos e metas, uma visão de Regras de Negócio precisa ser construída pelo analista. Para capturar estas regras, o ambiente fornece um interface com modelos de sentença $\mathrm{cm}$ português estruturado, o que permite que as regras sejam convertidas para o seu formato de armazenamento interno. Tal representação interna consiste de sentenças escritas na linguagem de programação em lógica PROLOG [Bratko 2000]. PROLOG baseia-se no subconjunto da lógica de primeira ordem composto pelas cláusulas de Horn [Hogger 1991]. Além da RN em si, várias informações adicionais podem ser cadastradas a seu respeito tais como autores, datas, fonte, motivação e relacionamentos, enriquecendo assim o conhecimento sobre o funcionamento da organização. A Figura 2 mostra a tela de definição das RNs do ambiente RÉGULA.

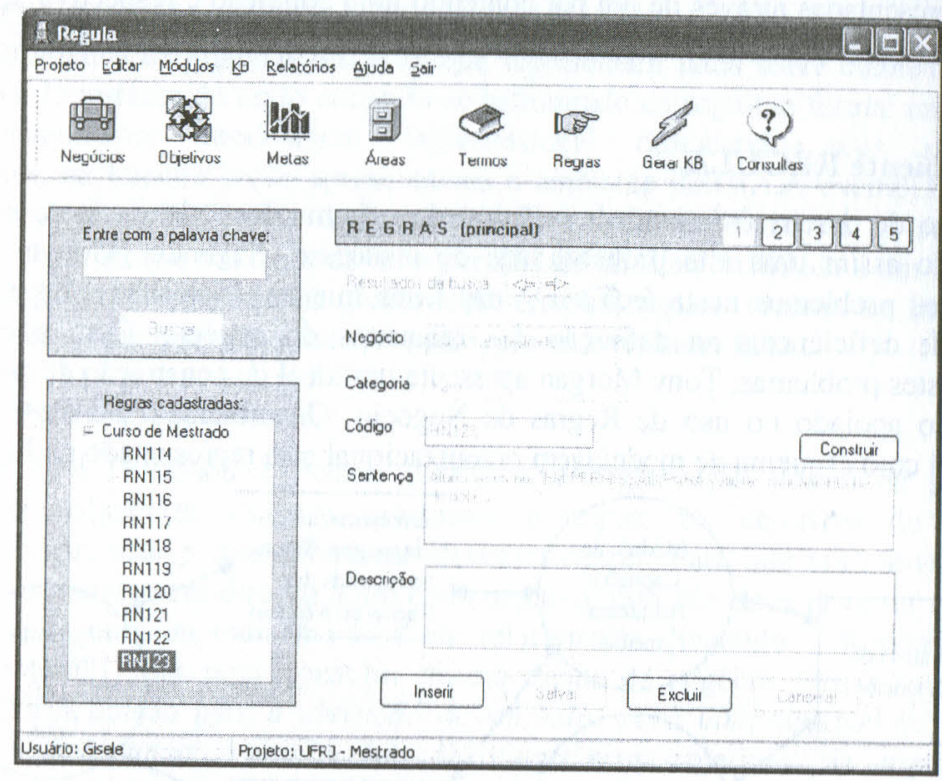

Figura 2. Interface de captura de Regras de Negócio.

As RNs usam os termos do negócio em sua construção. Os termos também são classificados como regras pelo [BRG 1997] e, mais atualmente, são referenciados como os componentes do vocabulário do negócio [Hall 2004]. Assim como acontece com as RNs, o RÉGULA provê uma forma de orientar o usuário na geração do vocabulário associado ao seu negócio. A formalização da definição do termo decompõe-se em três partes: relação de classificação (posicionamento na hierarquia de classes), finalidade $\mathrm{c}$ características. A relação de classificação é representada pela associação "é um subtipo de", definida sobre o termo em questão e um outro termo do vocabulário ou uma categoria básica. Utilizam-se as categorias básicas com o propósito de estabelecer um nivel mais genérico na relação de classificação. As cinco categorias básicas oferecidas dentro do RÉGULA baseiam-se naquelas definidas em [Shlacr e Mellor 1990]: evento, papel, relação de associação, especificação e coisa tangível. Finalidade é representada pela associação "serve para", vinculando o termo às regras as quais ele está relacionado.
As características do termo são representadas por relações deste com outros termos do vocabulário.

A interface de definição dos termos gera templates, que são mapeados para sentencas em PROLOG que representam formalmente o conceito A seguir, listamos os templates utilizados pelo RÉGULA: o template (1) representa a relação de classificação, o template (2) representa a de finalidade e os templates (3), (4) e (5) representam as características deste termo. Os templates (6) e (7) são utilizados na definição de um termo que possui relação de atributo de outro termo: o template (6) é a representação inversa do template (3) e o template (7) é utilizado para a definição dos possíveis valores de um atributo. Os termos que são atributos não possuem relação de finalidade nem outras características além do domínio.

$<$ Termo1>É SUBTIPO DE < Termo2:

$<$ Termol $>$ SERVE PARA $<$ RELAÇÃO $><$ Termo2

$<$ Termo 1> TEM COMO ATRIBUTO < Termo2>

$<$ Termol $>$ TEM COMO PARTE $<$ Termo2 $>$

$<$ Termo2 $>$ É ATRIBUTO DE $<$ Termo1 $>$

<Termo2 POSSUI COMO DOMÍNIO <Dominio

Para ilustrar a geração das sentenças em PROLOG a partir dos templates, utilizamos a definição do termo "candidato ao mestrado". Um candidato ao mestrado é definido como um subtipo de aluno que possui "coeficiente de rendimento acadêmico (cra)" aceitável e aprovação da "proposta de tese". A finalidade de um candidato é defender tese. O termo em questão possui ainda relação com o termo "tese" através do verbo "submeter" com grau 1 .. 1. As expressões (8) a (11) representam as cláusulas geradas para o processamento das informações referentes à definição do termo "candidato ao mestrado". Já a expressão (12) permite a consulta à definição do termo, assim como a geração do texto em linguagem natural que informa tal defínição ao usuário.

elemento(X, aluno) :- elemento(X, candidato_ao_mestrado)

elemento (X, candidato ao mestrado) :- possui (X, cra aceitavel),

foi_aprovado (X, proposta_de_tese)

finalidade ( $X$, candidato_ao_mestrado) :- defender ( $X$, tese)

submete $(X, Y, 1,1)$ : - elemento( $X$, candidato ao mestrado)

elemento( $Y$, proposta de tese)

definicaolcandidato_ao_mestrado, [aluno, [possui, cra_aceitavel],

[foi_aprovado, proposta_de_tese]], [[defender, tese]],

[ [submete, proposta de tese, 1,1$]]$ ).

O exemplo mostra que a captura do termo pelo RÉGULA também gera sentenças relativas a RNs, principalmente as do tipo "fato". Isto, aliado ao fornecimento da definição textual do termo para o usuário, facilita e reduz o trabalho de enunciado das RNs 


\subsection{Consulta a Permissões}

Uma vez capturados as regras e os termos do negócio, é possível utilizar o módulo responsável pela consulta a permissões do RÉGULA. Suponha que o analista de negócio digite uma pergunta em português estruturado. O módulo de consulta irá, em seguida, mapear a pergunta para a sua representação interna em PROLOG. Com o auxílio da hierarquia de classes e das permissões catalogadas na base de regras, a questão ć processada e o resultado obtido é traduzido de volta ao analista de negócio no formato textual padrão. Para que isso ocorra, as permissões já estão catalogadas pelo RÉGULA através dos templates (13) e (14), onde <Comp > é um operador de comparação (ex: maior, menor, igual, etc.) $\mathrm{e}<\mathrm{Qtd}>$ indica quantidade.

$<$ Termol $>$ TEM PERMISSÃO PARA $<$ Verbo $><$ Termo2 $>$

$<$ Termo1 $>$ TEM PERMISSÃO PARA $<$ Verbo $><$ Comp $><$ Qtd $><$ Termo2 $>$

A pergunta do usuário deve ser feita segundo um dos templates de consulta expressos por (15) a (19), onde FAZER indica que a consulta irá retornar, em caso de sucesso, um verbo ou expressão verbal. Similarmente, QUEM e O QUÊ resultarão, em caso de sucesso, um (ou mais) termo(s) catalogado(s). Os padrões de resposta para a consulta da expressão (13) possuem formato expresso por (20) e (21).

$<$ Termol $>$ TEM PERMISSÃO PARA $<$ Verbo $><$ Termo2 $>$ ?

QUEM TEM PERMISS ̃̃O PARA $<$ Verbo $><$ Termo2 $>$ ?

$<$ Termol $>$ TEM PERMISSÃO PARA < Verbo > O QUÊ?

$<$ Termol> TEM PERMISSÃO PARA FAZER O QUÊ?

QUEM TEM PERMISSÃO PARA FAZER OQUÊ?

RESP: SIM PELA REGRA <id regra>

RESP: Nก̃O.

Para ilustrar o funcionamento do módulo de consulta será utilizado o exemplo de um trancamento de disciplina simplificado. Considera-se que existam subtipos de "aluno", dentre eles o subtipo "aluno veterano"1. A representação interna dessa hierarquia é expressa por (22). Alunos têm permissão para solicitar o trancamento de disciplinas. Os termos, já capturados, e as permissões estão catalogados na base de regras. A representação interna da permissão em questão é expressa por (23).

elemento( $X$, aluno) :- elemento(X, aluno_veterano)

permissao(X, Y, solicitar, $r 1)$ :-

elemento(X, aluno), elemento(Y, trancamento_de_disciplina).

Considere uma consulta para verificar se aluno veterano tem permissão para solicitar trancamento de disciplina. A hierarquia de classes possibilita avaliar que os subtipos de "aluno" se enquadram nas permissões referentes a "aluno", em especial "aluno veterano" herda a permissão concedida a "aluno" para "solicitar" "trancamento de disciplina". A expressão (24) mostra a consulta, que utiliza o template (15), referente à expressão (13), cuja resposta é dada por (25). aluno_veterano TEM PERMISSÃO PARA solicitar trancamento_de_disciplina? RESP: SIM PELA REGRA rl.

Cada consulta fornece, além da resposta, o número da RN que identifica a permissão em questão (se ela existir). Caso a implementação deste módulo fosse realizada por um banco de dados relacional comercial, a representação da herança de permissões pela hierarquia de classes de termos só seria possivel através da catalogação de pares não genéricos de nós pai-filho.

\section{Conclusão}

Este artigo apresentou uma abordagem para Modelagem Organizacional baseada em RNs. Esta abordagem é viabilizada pelo ambiente RÉGULA que está inserido no contexto da Bancada Virtual para Projetos de Negócio [Schmitz et al 2004]. O uso de tal plataforma representa um avanço no sentido de tornar mais eficiente a gestão do a de análise e do levantamento de requisitos relativos às Regras de Negócio, facilitando o desenvolvimento de sistemas de informação

A utilização da linguagem da lógica de primeira ordem (LPO) como forma interna de armazenamento provê formalismo sólido para representação e processamento das RNs. Através da LPO torna-se possível verificar a consistência das RNs e realizar consultas sobre um determinado subconjunto de suas sentenças onde se pode determinar computacional. Convém ressaltar que não apenas o subconjunto da LPO composto pelas cláusulas de Horn constitui base teórica para a representação de RNs mas também lógicas não-clássicas como a lógica modal (deôntica, alética, temporal, etc.) [Chellas 1980] [Meyer, Wieringa e Dignum 1998] [Gabbay 1994], além de outras formas de raciocínio não-monotônico [Genesereth e Nilsson 1987]. O uso dos templates em português estruturado para definição de termos e demais RNs e para entrada e saída do módulo de consulta a permissões, obrigações proibições torna natural e acessível ao analista de negócio a modelagem da organização.

$\mathrm{O}$ enunciado de objetivos e metas integrado ao das RNs e a captura organizada dos termos constitui funcionalidade inovadora em relação a sistemas de gerência e armazenamento de RNs [BRS 2004]. Além disso, estudos preliminares sugerem a ausência de referências bibliográficas a sistemas que permitam a consulta automatizada a permissões, obrigações e proibições e a captura dirigida dos termos. Outros trabalhos correlatos somente deverão ser divulgados após a conclusão da apreciação da OMG sobre propostas para a definição semântica de RNs [OMG 2004].

Quanto à verificação na prática dos benefícios da abordagem aqui apresentada, empresas estão sendo contatadas para a realização de estudos de caso. Também se empresto de caso interno envolvendo o regulamento do mestrado do NCE. Outros trabalhos em desenvolvimento visam ao aumento da integração do RÉGULA com o restante da Bancada Virtual, em particular com a ferramenta de geração de casos de uso a partir dos diagramas de atividades chamada HPReq [Cruz 2004], e com o módulo verificador de processos. Dentre os tópicos de pesquisa em um futuro próximo podemos citar a geração automática do modelo de classes de domínio e a verificação parcial de consistência das RNs, especialmente da consistência entre permissões, obrigações e proibições. 


\section{Referências Bibliográficas}

Bratko, I. (2000) PROLOG Programming for Artificial Intelligence, Addison Wesley.

BRG (1997) "BUSINESS Rules Group, GUIDE Business Rules Project: Final Report", revisão 1.2 .

BRG (2000) "BUSINESS Rules Group, Defining Business Rules _ What Are They Really?", revisão 1.3 .

BRS (2004) "RuleTrack", Business Rules Solutions, URL: http://www brsolutions.com/, acessado em maio/2004.

Chellas, B. (1980) Modal Logic: An Introduction, Cambridge University Press.

Cruz, P. O. S. (2004) "Heurísticas para Identificação de Requisitos de Sistemas de Informações a partir de Modelos de Processos", UFRJ/IM/NCE

Eriksson, H-E. e M. Penker, (2000) Business Modeling with UML: Business Patterns at Work, John Wiley \& Sons.

Genesereth, e Nilsson, (1987) Logical Foundations of Artificial Intelligence, Morgan Kaufmann.

Gabbay, D., (1994) Temporal Logic: Mathematical Foundations and Computational Aspects, Oxford University Press.

Hall, J. (2()04) "Business Semantics of Business Rules," Business Rules Journal, Vol. 5, No. 3 (Mar. 2004), URL: http://www.BRCommunity.com/a2004/b 182.html

Hogger, C. J. (1994) Essentials of Logic Programming, Oxford University Press.

Koubarakis, M. e Plexousakis, D. (2002) "A Formal Framework for Business Process Modeling and Design, Information Systems", 27 299-319, Elsevier.

Meyer, J-J., R. Wieringa e F. Dignum. (1998) "The Role of Deontic Logic in the Specification of Information Systems", Logics for Databases and Information Systems, 71-115.

Morgan, T. (2002) "Business Rules and Information Systems - Aligning IT with Business Goals". Addison-Wesley.

OMG (2004) "Business Semantics of Business Rules Request For Proposal". http://www.omg.org/docs/br/03-03-03.pdf

Ross, R. G. (2000) "What is a Business Rule?". Disponivel em http://www.brcommunity.com/cgi-bin/x.pl/commentary/b005.html. Acessado em: 04 de Junho de 2004.

Schmitz, E. A., Lima, P. M. V., Silveira, D. S., Seabra, C. M., Martins, A. E., Morgado, G. P., Dias, F. G. (2004) "Uma Infra-Estrutura Virtual para Projetos de Empresas" Relatório Técnico, UFRJ/IM/NCE, Agosto.

Shlaer, S. c Mellor, S. (1990) "Análise de Sistemas Orientada para Objetos".McGrawHill. 